\title{
Some properties of two-fuzzy Nor med spaces
}

\author{
Noori F.AL-Mayahi, Layth S.Ibrahaim \\ ${ }^{1}$ (Department of Mathematics, College of Computer Science and Mathematics, University of AL -Qadissiya) \\ ${ }^{2}$ (Department of Mathematics, College of Computer Science and Mathematics, University of AL-Qadissiya)
}

Abstract: The study sheds light on the two-fuzzy normed space concentrating on some of their properties like convergence, continuity and the in order to study the relationship between these spaces

Keywords: fuzzy set, Two-fuzzy normed space, $\alpha$-norm,

2010 MSC: $46 \mathrm{~S} 40$

\section{Introduction}

The concept of fuzzy set was introduced by Zadeh [2] in 1965 as an extension of the classical notion of set. A satisfactory theory of 2-norm on a linear space has been introduced and developed by Gahler in [4]. The concept of fuzzy norm and $\alpha$-norm were introduced by Bag and Samanta and the notions of convergent and Cauchy sequences were also discussed in [6]. zhang [1] has defined fuzzy linear space in a different way. RM. Somasundaram and ThangarajBeaula defined the notion of fuzzy 2-normed linear space $(F(X) ; N)$ or 2- fuzzy 2normed linear space. Some standard results in fuzzy 2 - normed linear spaces were extended. The famous closed graph theorem and Riesz Theorem were also established in 2-fuzzy 2-normed linear space. In [5], we have introduced the new concept of 2-fuzzy inner product space on $F(X)$, the set of all fuzzy sets of $X$. This paper is about the concepts related to two-fuzzy normed spaces (fuzzy convergence and fuzzy continuity).

\section{PRELIMINARIES}

Definition2.1.[3]Let $X$ be a real vector space of dimension greater than one and let $\|.,$.$\| be a real valued$ function on $\mathrm{X} \times \mathrm{X}$ satisfying the following conditions:

(1) $\|x, y\|=0$ if and only if $\mathrm{x}$ and $\mathrm{y}$ are linearly dependent,

(2) $\quad\|x, y\|=\|y, x\|$

(3) $\|\alpha x, y\|=|a|\|x, y\|$ where $\alpha$ is real,

(4) $\quad\|x, y+z\|<\|x, y\|+\|x, z\|$

$\|.,$.$\| is called a two-norm on X$ and the pair $(X,\|.,\|$.$) is called a two normed liner space.$

Definition2.2.[3]Let $X$ be a vector space over $\mathrm{K}$ (the field of real or complex numbers). A fuzzy subset $\mathrm{N}$ of $X \times \mathbb{R}$ (R, the set of real numbers) is called a fuzzy norm on $\mathrm{X}$ if and only if for all

(N1) For all $t \in \mathbb{R}$ with $t \leq 0, N(x, t)=0$

$$
x, y \in X \text { and } c \in K \text {. }
$$

(N2) For all $t \in \mathbb{R}$ with $t>0, N(x, t)=1$ if and only if $x=0$

(N3) For all $t \in \mathbb{R}$ with $t>0, N(c x, t)=N(x, C)=N\left(x, \frac{x}{|c|}\right)$, if $c=0$

(N4) For all $s, t \in \mathbb{R}, x, y \in X, N(f+g, s+t)>\min \{N(x, s), N(y, t)\}$

(N5) $N\left(x\right.$, . ) is a non decreasing function of $\mathbb{R}$ and $\lim _{\mathrm{t} \rightarrow \infty} \mathrm{N}(\mathrm{x}, \mathrm{t})=1$

The pair $(X, N)$ will be referred to be as a fuzzy normed linear space.

Theorem2.3.[3]Let $(X, N)$ be a fuzzy normed linear space . Assume further that $(N 6) N(x, t)>$ 0 for all $t>0$ implies $x=0$.

Define $\|x\|_{\alpha}=\inf \{t: N(x, t)>\alpha\}$ where $\alpha \in(0,1)$.

Then $\left\{\|x\|_{\alpha}: \alpha \in(0,1)\right\}$ is an ascending family of norms on $X$ (or) $\alpha$-norms on $X$ corresponding to the fuzzy norm on $X$.

Definition2.4.[3]A fuzzy vector space $\tilde{X}=X \times(0,1]$ over the number field $\mathrm{K}$, where the addition and scalar multiplication operation on $X$ are defined by

$$
(x, \lambda)+(y, \mu)=(x+y, \lambda \wedge \mu) \text { and } k(x, \lambda)=(k x, \lambda)
$$

is a fuzzy normed space if to every $(x, \lambda) \in \tilde{X}$ there is associated a non-negative real number, $\|(x, \lambda)\|$, called the fuzzy norm of $(x, \lambda)$, in such a way that

$$
\|(x, \lambda)\|=0 \text { iff } x=0 \text { the zero element of } X, \lambda \in(0,1]
$$


(2) $\quad\|(k x, \lambda)\|=|k|\|(x, \lambda)\|$, for all $(x, \lambda) \in \widetilde{X}$ and all $k G K$

(3) $\quad\|(x, \lambda)+(y, \mu)\|<\|(x, \lambda \wedge \mu)\|+\|(y, \lambda \wedge \mu)\|$ for all $(x, \lambda)$ and

$$
(y, \mu) \in \widetilde{X}
$$

(4) $\quad\left\|U\left(x, V \lambda_{t}\right)\right\|=V\left\|\left(x, \lambda_{t}\right)\right\|$ for $\lambda_{t} \in(0,1]$

Let $X$ be a nonempty and $F(X)$ be the set of all fuzzy sets in X. If

$f \in F(X)$ then $f=\{(x, \mu): x \in X$ and $\mu \in(0,1]\}$. Clearly $\mathrm{f}$ is a bounded

function for $|f(x)| \leq 1$. Let $\mathrm{K}$ be the space of real numbers, then $\mathrm{F}(\mathrm{X})$ is a linear space over the field $\mathrm{K}$ where the addition and scalar multiplication are defined by

$$
f+g=\{(x, \mu)+(y, \eta)\}=\{(x+v, \mu \wedge \eta):(x, \mu) \in f, \text { and }(y, n) \in g\}
$$

$k f=\{(k f, \mu):(x, \mu) \in f\}$ where $k \in K$. The linear space $F(X)$ is said to be normed space if to every $f \in$ $F(X)$, there is associated a non-negative real number $\|f\|$ called the norm of $\mathrm{f}$ in such a way that

$$
\begin{gathered}
\|f\|=0 \text { if and only if } f=0 \text { For, } \\
\qquad \begin{array}{l}
\|f\|=0 \Leftrightarrow\{\|x, \mu\|:(x, \mu) \in f\}=0 \\
\Leftrightarrow x=0, \quad \mu \in(0,1] \\
\Leftrightarrow f=0 .
\end{array}
\end{gathered}
$$

(2) $\quad\|k f\|=|k|\|f\|, k \in K \quad$ For,

$\|k f\|=\{\|k x, \mu\|:(x, \mu) \in f, k \in K\}$

$=\{|k|\|x, \mu\|:(x, \mu) \in f\}=|k|\|f\|$

(3) $\quad\|f+g\| \leq|f|+|g|$ for every $f, g \in F(X)$

For,$\|f+g\|=\{\|(x, \mu)+(y, \eta)\| / x, y \in X, \mu, \eta \in(0,1]\}$

$$
\begin{gathered}
=\{\|(x+y), \mu \wedge \eta\|: x, y \in X, \mu, \eta \in(0,1]\} \\
\leq\{\|x, \mu\|+\|y, \mu \wedge \eta\|:(x, \mu) \in \text { fand }(y, \eta) \in g\} \\
=\|f\|+\|g\|
\end{gathered}
$$

And so $(F(X),\|\|$.$) is a normed linear space.$

Definition2.6.[3]Let $X$ be any non-empty set and $F(X)$ be the set of all fuzzy sets on $X$. Then for $U, V \in$ $F(X)$ and $k \in K$ the field of real numbers, define

$k U=\{(k x, \lambda):(x, \lambda) \in U\}$.

$$
U+V=\{(x+y, \lambda \Lambda \mu):(x, \lambda) \in U,(y, \mu) \in V\} \text { and }
$$

Definition2.7.[3]A two-fuzzy set on $X$ is a fuzzy set on $F(X)$.

Definition2.8.[3]Let $\mathrm{F}(\mathrm{X})$ be a vector space over the real field $\mathrm{K}$. A fuzzy subset $\mathrm{N}$ of

$F(X) \times \mathbb{R}$, $(\mathbb{R}$, the set of real numbers) is called a 2-fuzzy norm on $F(X)$ if and only if,

(N1) For all $t \in \mathbb{R}$ with $t \leq 0, N(f, t)=0$

(N2) For all $t \in \mathbb{R}$ with $t>0, N(f, t)=1$ if and only if $f=0$

(N3) For all $t \in \mathbb{R}$, with $t \geq 0, N(c f, t)=N\left(f, \frac{y}{|c|}\right)$ if $c \neq 0, c \in K$ (field)

(N4) For all $s, t \in \mathbb{R}, N\left(f_{1}+f_{2}, s, t\right) \geq \min \left\{N\left(f_{1}, s\right), N\left(f_{2}, t\right)\right\}$

(N5) $N(f, \bullet):(0, \infty) \rightarrow[0,1]$ is continuous

(N6) $\lim _{t \rightarrow \infty} N(f, t)=1$

Then the pair $(F(X), N)$ is a fuzzy two-normed vector space.

\section{Mainresult}

Definition 3.1.Let $(F(X), N, *)$ be a two-fuzzy normed space, then:

(a) A sequence $\left\{f_{n}\right\}$ in $F(X)$ is said to fuzzy converges to $f$ in $F(X)$ if for each $\varepsilon \in(0,1)$ and each $t>0$, there exists $n_{0} \in Z^{+}$such that $N\left(f_{n}-f, t\right)>1-\varepsilon \quad$ for all $n \geq n_{0}($ Or equivalently $\left.\lim _{n \rightarrow \infty} N\left(f_{n}-f, t\right)=1\right)$.

(b) A sequence $\left\{f_{n}\right\}$ in $F(X)$ is said to be fuzzy Cauchy if for each $\varepsilon \in(0,1)$ and each $t>0$, there exists $n_{0} \in Z^{+}$such that $N\left(f_{n}-f_{m}, t\right)>1-\varepsilon$ for all $n, m \geq n_{0}$ (Or equivalently

$$
\left.\lim _{n, m \rightarrow \infty} N\left(f_{n}-f_{m}, t\right)=1\right)
$$

(c) A two-fuzzy normed space in which every fuzzy Cauchy sequence is fuzzy convergent is said to be complete.

Theorem 3.2. Let $(F(X), N, *)$ be a two-fuzzy normed space and let $\left\{f_{n}\right\},\left\{g_{n}\right\}$ be two sequences in two-fuzzy normed space $F(X)$, and for all $\alpha_{1} \in(0,1)$ there exist $\alpha \in(0,1)$ such that $\alpha * \alpha \geq \alpha_{1}$.

(1) Every fuzzy convergent sequence is fuzzy Cauchy sequence. 
(2) Every sequence in $F(X)$ has a unique fuzzy limit.

(3) If $f_{n} \rightarrow f$ then $c f_{n} \rightarrow c f, c \in \mathbb{F} /\{0\}$.

(4) If $f_{n} \rightarrow f, g_{n} \rightarrow g$, then $f_{n}+g_{n} \rightarrow f+g$.

Proof: (1) Let $\left\{f_{n}\right\}$ be a sequence in $F(X)$ such that $f_{n} \rightarrow f$ then for all $t>0, \lim _{n \rightarrow \infty} N\left(f_{n}-f, \frac{t}{2}\right)=1$,

$N\left(f_{n}-f_{m}, t\right)=N\left(\left(f_{n}-f\right)-\left(f_{m}-f\right), t\right) \geq N\left(f_{n}-f, \frac{t}{2}\right) * N\left(f_{m}-f, \frac{t}{2}\right)$, by taking $\operatorname{limit}_{\lim } \lim _{n \rightarrow \infty} N\left(f_{n}-\right.$ $f m, t \geq \lim n \rightarrow \infty N f n-f, t 2 * \lim m \rightarrow \infty N f m^{-} f, t 2=1 * 1=1 \quad$ but $\quad \lim n, m \rightarrow \infty N f n-f m, t \leq 1$ then $\lim _{n, m \rightarrow \infty} N\left(f_{n}-f_{m}, t\right)=1$ therefore $\left\{f_{n}\right\}$ is a Cauchy sequence in $F(X)$.

(2) Let $\left\{f_{n}\right\}$ be a sequence in $F(X)$ such that $f_{n} \rightarrow f$ and $f_{n} \rightarrow g$ as $n \rightarrow \infty$ and $f \neq g$ then for all $t>s>0$, $\lim _{n \rightarrow \infty} N\left(f_{n}-f, s\right)=1, \lim _{n \rightarrow \infty} N\left(f_{n}-g, t-s\right)=1 N(f-g, t) \geq N\left(f_{n}-f, s\right) * N\left(f_{n}-g, t-s\right)$

Taking limit as $n \rightarrow \infty$ :

$N(f-g, t) \geq 1 * 1=1 . \Rightarrow \operatorname{but} N(f-g, t) \leq 1 \Rightarrow N(f-g, t)=1$.

Then by axiom (ii) $f-g=0 \Rightarrow f=g$.

(3) Since $f_{n} \rightarrow f$ then if for all $\varepsilon \in(0,1)$ and for all $t>0$, there exists

$n_{0} \in Z^{+}$such that $N\left(f_{n}-f, t\right)>1-\varepsilon$ for all $n \geq n_{0}$ put $t=\frac{t 1}{|c|}$.

$N\left(c f_{n}-c f, t_{1}\right)=N\left(f_{n}-f, \frac{t 1}{|c|}\right)=N\left(f_{n}-f, t\right)>1-\varepsilon$

Then $c f_{n} \rightarrow c f$.

(4) For each $\varepsilon_{1} \in(0,1)$ there exists $\varepsilon \in(0,1)$ such that $\quad(1-\varepsilon) *(1-\varepsilon) \geq\left(1-\varepsilon_{1}\right)$. Since $x_{n} \rightarrow x$ then for each $\varepsilon \in(0,1)$ and each $t>0$, there exists $n_{1} \in Z^{+}$such that $N\left(f_{n}-f, \frac{t}{2}\right)>1-\varepsilon$ for all

$n \geq n_{1}$, since $g_{n} \rightarrow g$ then if for each $\varepsilon_{\in(0,1)}$ and each $t>0$, there exists $n_{2} \in Z^{+}$such that

$N\left(g_{n}-g, \frac{t}{2}\right)>1-\varepsilon$ for all $n \geq n_{2}$. Take $n_{0}=\min \left\{n_{1}, n_{2}\right\}$, and for each $t>0$, there exists $n_{0} \in Z^{+}$such that

$N\left(\left(f_{n}+g_{n}\right)-(f+g), t\right)=N\left(\left(f_{n}-f\right)+\left(g_{n}-g\right), t\right) \geq$

$N\left(f_{n}-f, \frac{t}{2}\right) * N\left(g_{n}-g, \frac{t}{2}\right)>(1-\varepsilon) *(1-\varepsilon) \geq\left(1-\varepsilon_{1}\right)$ for all $n \geq n_{0}$. Then $f_{n}+g_{n} \rightarrow f+g$.

Theorem 3.3.Let $(F(X), N, *),(F(Y), N, *)$ be a two-fuzzy normed spaces and let $f_{n} \rightarrow f, g_{n} \rightarrow g$, such that $\left\{f_{n}\right\}$ and $\left\{g_{n}\right\}$ are two sequences in $F(X)$ and $\alpha, \beta \in \mathbb{F} /\{0\}$ then $\alpha \Psi\left(f_{n}\right)+\beta \omega\left(g_{n}\right) \rightarrow \alpha \Psi(f)+\beta \omega(g)$ whenever $\psi$ and $\omega$ are two identity fuzzy functions.

Proof: For all $\varepsilon \in(0,1)$ there exist $\varepsilon_{1} \in(0,1)$ such that

$f_{n} \rightarrow f$, then for all $\varepsilon_{1} \in(0,1)$ and $t>0$ there exists $n_{1} \in Z^{+}$such that

$\left(1-\varepsilon_{1}\right) *\left(1-\varepsilon_{1}\right) \geq(1-\varepsilon)$, since $N\left(f_{n}-f, \frac{t}{2|\alpha|}\right)>\left(1-\varepsilon_{1}\right)$ for all $n \geq n_{1}$, and since $g_{n} \rightarrow g$ then for all $\varepsilon_{1} \in(0,1)$ and $\mathrm{t}>0$ there exists $n_{2} \in Z^{+}$such that $N\left(g_{n}-g, \frac{t}{2|\beta|}\right)>\left(1-\varepsilon_{1}\right)$ for all $n \geq n_{2}$. Take $n_{0}=\min \left\{n_{1}, n_{2}\right\}, n \geq n_{0}$

$N\left(\left(\alpha \psi\left(f_{n}\right) \quad+\beta \omega\left(g_{n}\right)\right)-(\alpha \psi(f)+\beta \omega(g)), t\right)=\quad N\left(\alpha \alpha\left(\psi\left(f_{n}\right)-\psi(f)\right)+\beta\left(\omega\left(g_{n}\right)-\omega(g)\right), t\right) \geq$ $N\left(\Psi\left(f_{n}\right)-\Psi(f), \frac{t}{2|\alpha|}\right) * N\left(\omega\left(g_{n}\right)-\omega(g), \frac{t}{2|\beta|}\right)$

$=N\left(f_{n}-f, \frac{t}{2|\alpha|}\right) * N\left(g_{n}-g, \frac{t}{2|\beta|}\right)>\left(1-\varepsilon_{1}\right) *\left(1-\varepsilon_{1}\right) \geq(1-\varepsilon)$

$\alpha \Psi\left(f_{n}\right)+\beta \omega\left(g_{n}\right) \rightarrow \alpha \psi(f)+\beta \omega(g)$.

Theorem 3.4. A two-fuzzy normed space $(F(X), N, *)$ is complete two-fuzzynormed space if every fuzzy Cauchy sequence $\left\{f_{n}\right\}$ in $F(X)$ has a fuzzy convergent subsequence.

Proof: Let $\left\{f_{n}\right\}$ be a fuzzy Cauchy sequence in $F(X)$ and $\left\{f_{n m}\right\}$ be a subsequence of $\left\{f_{n}\right\}$ such that $f_{n m} \rightarrow f$, $f \in F(X)$.

Now to prove $f_{n} \rightarrow f$. For all $\varepsilon \in(0,1)$ there exist $\varepsilon_{1} \in(0,1)$ such that $\left(1-\varepsilon_{1}\right) *\left(1-\varepsilon_{1}\right) \geq(1-\varepsilon)$. Since $\left\{f_{n}\right\}$ is a fuzzy Cauchy sequence then for all $t>0$ and $\varepsilon_{1} \in(0,1)$ there exists $n_{0} \in Z^{+}$such that: $N\left(f_{n}-f_{m}, \frac{t}{2}\right)>$ $1-\varepsilon_{1}$, for all $n, m \geq n_{0}$.

Since $\left\{f_{n m}\right\}$ is fuzzy convergent to $f$, there exists $\mathrm{im} \geq n_{0}$ such that $N\left(f_{\text {im }}-f, \frac{t}{2}\right)>1-\varepsilon_{1}$

$N\left(f_{n}-f, \mathrm{t}\right)=N\left(\left(f_{n}-f_{i m}\right)+\left(f_{i m}-x\right), \frac{t}{2}+\frac{t}{2}\right) \geq$

$N\left(x_{n}-x_{i m}, \frac{t}{2}\right) * N\left(f_{i m}-f, \frac{t}{2}\right)>\left(1-\varepsilon_{1}\right) *\left(1-\varepsilon_{1}\right) \geq(1-\varepsilon)$.

Therefore $f_{n} \rightarrow f,\left\{f_{n}\right\}$ is fuzzy convergent to $f$ Hence $(F(X), N, *)$ is complete two-fuzzy normed space. 
Definition 3.5. Let $(F(X), N, *)$ and $(F(Y), N, *)$ be two-fuzzy normed spaces. The function $\Psi: F(X) \rightarrow F(Y)$ is said to be fuzzy continuous at $f_{0} \in F(X)$ if for all $\varepsilon \in(0,1)$ and all $t>0$ there exist $\delta \in(0,1)$ and $s>0$ such that for all $f \in F(X)$

$N\left(f-f_{0}, s\right)>1-\delta$ implies $N\left(\Psi(f)-\psi\left(f_{0}\right), t\right)>1-\varepsilon$.

The function $f$ is called a fuzzy continuous function if it is fuzzy continuous at every point of $F(X)$.

Theorem 3.6. Every identity fuzzy function is fuzzy continuous function in two-fuzzy normed space.

Proof: For all $\varepsilon \in(0,1)$ and $t>0$ there exist $s=t$ and $<\varepsilon, \delta \in(0,1), \quad N\left(f_{n}-f, s\right)>1-\delta$

$N\left(\psi\left(f_{n}\right)-\psi(f), t\right)=N\left(f_{n}-f, s\right)>1-\delta>1-\varepsilon$ therefore $\psi$ is a fuzzy continuous at $f$, since $f$ is an arbitrary point then $\psi$ is a fuzzy continuous function.

Theorem 3.7.Let $F(X)$ be a two-fuzzy normed space over $\mathbb{F}$. Then the functions $\quad$ : $F(X) \times F(X) \rightarrow$ $F(X), \psi(f, g)=f+g$ and

$\omega: \mathbb{F} \times F(X) \rightarrow F(X), \omega(\lambda, f)=\lambda f$ are fuzzy continuous functions.

Proof: (1) Let $\varepsilon \in(0,1)$ then there exists $\varepsilon_{1} \in(0,1)$ such that

$\left(1-\varepsilon_{1}\right) *\left(1-\varepsilon_{1}\right) \geq(1-\varepsilon)$.

let $f, g \in F(X)$ and $\left\{f_{n}\right\},\left\{g_{n}\right\}$ in $F(X)$ such that $f_{n} \rightarrow f$ and $g_{n} \rightarrow g$ as $n \rightarrow \infty$, then for each $\varepsilon_{1} \in(0,1)$ and each $\frac{t}{2}>0$ there exists $n_{1} \in Z^{+}$such that $N\left(f_{n}-f, \frac{t}{2}\right)>1-\varepsilon_{1}$ for all $n \geq n_{1}$, and for each $\varepsilon_{1}>0$ and $\frac{t}{2}>0$ there exists $n_{2} \in Z^{+}$such that $N\left(g_{n}-g, \frac{t}{2}\right)>1-\varepsilon_{1}$ for all $n \geq n_{2}$, put $n_{0}=\min \left\{n_{1}, n_{2}\right\} N\left(f\left(x_{n}, y_{n}\right)-f(x, y), t\right)=N\left(\left(f_{n}+g_{n}\right)-(f+g), t\right)=N\left(\left(f_{n}-f\right)+\left(g_{n}-g\right), t\right) \geq$ $N\left(f_{n}-f, \frac{t}{2}\right) * N\left(g_{n}-g, \frac{t}{2}\right)>\left(1-\varepsilon_{1}\right) *\left(1-\varepsilon_{1}\right) \geq 1-\varepsilon$ for all $n \geq n_{0}$, therefore $\quad \psi\left(f_{n}, g_{n}\right) \rightarrow$ $\psi(f, g)$ as $n \rightarrow \infty, \psi$ is fuzzy continuous function at $(x, y)$ and $(x, y)$ is any point in $F(X) \times F(X)$, hence $\psi$ is fuzzy continuous function.

(2) Let $f \in F(X), \lambda \in \mathbb{F}$ and $\left\{f_{n}\right\}$ in $F(X),\left\{\lambda_{n}\right\}$ in $\mathbb{F}$ such that $f_{n} \rightarrow f$ and $\lambda_{n} \rightarrow \lambda$ as $n \rightarrow \infty$, then for each $\frac{t}{2\left|\lambda_{n}\right|}>0, \lim _{n \rightarrow \infty} N\left(f_{n}-f, \frac{t}{2\left|\lambda_{n}\right|}\right)=1,\left|\lambda_{n}-\lambda\right| \rightarrow 0$ as $n \rightarrow \infty$,

$N\left(\omega\left(\lambda_{n}, f_{n}\right)-\omega(\lambda, f), t\right)=N\left(\lambda_{n} f_{n}-\lambda f, t\right)=$ $f \lambda), t) \geq N\left(\lambda_{n}\left(f_{n}-f\right), \frac{t}{2}\right) * N\left(f\left(\lambda_{n}-\lambda\right), \frac{t}{2}\right)=N\left(f_{n}-f, \frac{t}{2\left|\lambda_{n}\right|}\right) * N\left(f, \frac{t}{2\left|\lambda_{n}-\lambda\right|}\right), \quad$ by taking limit: $\lim _{n \rightarrow \infty} N\left(\omega\left(\lambda_{n}, f_{n}\right)-\omega(\lambda, f), t\right) \geq \lim _{n \rightarrow \infty} N\left(f_{n}-f, \frac{t}{2\left|\lambda_{n}\right|}\right) * \lim _{n \rightarrow \infty} N\left(f, \frac{t}{2\left|\lambda_{n}-\lambda\right|}\right)=1 * 1=1 \quad$ but $\lim _{n \rightarrow \infty} N\left(\omega\left(\lambda_{n}, f_{n}\right)-\omega(\lambda, f), t\right) \leq 1$ then $\lim _{n \rightarrow \infty} N\left(\omega\left(\lambda_{n}, f_{n}\right)-\omega(\lambda, f), t\right)=1$ then

$\omega\left(\lambda_{n}, f_{n}\right) \rightarrow \omega(\lambda, f)$ as $n \rightarrow \infty, \omega$ is fuzzy continuous at $(\lambda, \omega)$ and $(\lambda, f)$ is any point in $\mathbb{F} \times F(X)$, hence $\omega$ is fuzzy continuous.

Theorem 3.8. Let $(F(X), N, *)$ and $(F(Y), N, *)$ be two-fuzzy normed spaces and let $\psi: F(X) \rightarrow F(Y)$ be a linear function. Then $\psi$ is a fuzzy continuous either at every point of $F(X)$ or at no point of $F(X)$.

Proof: Let $f_{1}$ and $f_{2}$ be any two points of $F(X)$ and suppose $\psi$ is fuzzy continuous at $f_{1}$. Then for each $\varepsilon \in(0,1)$, $t>0$ there exist $\delta \in(0,1)$ such that $f \in F(X), N\left(f-f_{1}, s\right)>1-\delta \Rightarrow N\left(\psi(f)-\psi\left(f_{1}\right), t\right)>1-\varepsilon$ Now: $N\left(f-f_{2}, s\right)>1-\delta, N\left(\left(f+f_{1}-f_{2}\right)-f_{1}, \quad s\right)>1-\delta \Rightarrow N\left(\Psi\left(f+f_{1}-f_{2}\right)-\psi\left(f_{1}\right), t\right)>1-\varepsilon \Rightarrow$ $N\left(\Psi(f)+\psi\left(f_{1}\right)-\psi\left(f_{2}\right)-\psi\left(f_{1}\right), t\right)>1-\varepsilon \Rightarrow N\left(\Psi(f)-\psi\left(f_{2}\right), t\right)>1-\varepsilon, \psi$ is a fuzzy continuous at $f_{1}$, since $f_{2}$ is arbitrary point. Hence $\psi$ is a fuzzy continuous.

Corollary 3.9.Let $(F(X), N, *)$ and $(F(Y), N, *)$ be two-fuzzy normed spaces and let $\Psi: F(X) \rightarrow F(Y)$ be a linear function. If $\psi$ is fuzzy continuous at 0 then it is fuzzy continuous at every point.

Proof: Let $\left\{f_{n}\right\}$ be a sequence in $F(X)$ such that there exist $f_{0}$, and $f_{n} \rightarrow f_{0}$, since $\psi$ is fuzzy continuous at 0 then: For all $\varepsilon \in(0,1), t>0$ there exist $\delta \in(0,1), s>0:\left(f_{n}-f_{0}\right) \in F(X)$

,$N\left(\left(f_{n}-f_{0}\right)-0, s\right)>1-\delta \Rightarrow N\left(\Psi\left(f_{n}-f_{0}\right)-\Psi(0), t\right)>1-\varepsilon$,

$N\left(f_{n}-f_{0}, s\right)>1-\delta \Rightarrow N\left(\Psi\left(f_{n}\right)-\psi\left(f_{0}\right)-\psi(0), t\right)>1-\varepsilon$

$N\left(f_{n}-f_{0}, s\right)>1-\delta \Rightarrow N\left(\Psi\left(f_{n}\right)-\psi\left(f_{0}\right)-0, t\right)>1-\varepsilon$

$N\left(f_{n}-f_{0}, s\right)>1-\delta \Rightarrow N\left(\Psi\left(f_{n}\right)-\psi\left(f_{0}\right), t\right)>1-\varepsilon$

$f_{n} \rightarrow f_{0} \Rightarrow \Psi\left(f_{n}\right) \rightarrow \Psi\left(f_{0}\right)$ therefore $\Psi$ is fuzzy continuous at $f_{0}$ since $f_{0}$ is arbitrary point, then $\Psi$ is fuzzy continuous function.

Theorem 3.10.Let $(F(X), N, *),(F(Y), N, *)$ be a two-fuzzy normed spaces, then the function $\psi: F(X) \rightarrow F(Y)$ is fuzzy continuous at $f_{0} \in F(X)$ if and only if for all fuzzy sequence $\left\{f_{n}\right\}$ fuzzy convergent to $f_{0}$ in $X$ then the sequence $\left\{\psi\left(f_{n}\right)\right\}$ is fuzzy convergent to $\Psi\left(f_{0}\right)$ in $Y$.

Proof: Suppose the function $\Psi$ is fuzzy continuous in $f_{0}$ and let $\left\{f_{n}\right\}$ is a sequence in $F(X)$ such that $f_{n} \rightarrow f_{0}$. Let $\varepsilon \in(0,1), t>0$, since $\psi$ is fuzzy continuous in $f_{0} \Rightarrow$ there exist $\delta \in(0,1), s>0$, such that for all 
$f \in F(X): N\left(f-f_{0}, s\right)>1-\delta \Rightarrow N\left(\psi(f)-\psi\left(f_{0}\right), t\right)>1-\varepsilon$

Since $f_{n} \rightarrow f_{0}, \delta \in(0,1), s>0$, there exist $k \in Z^{+}$such that

$N\left(f_{n}-f_{0}, s\right)>1-\delta$ for all $n \geq k$ hence $N\left(\psi\left(f_{n}\right)-\psi\left(f_{0}\right), t\right)>1-\varepsilon$ for all $n \geq k$ therefore $\psi\left(f_{n}\right) \rightarrow \psi\left(f_{0}\right)$.

Conversely suppose the condition in the theorem is true.

Suppose $\psi$ is not fuzzy continuous at $f_{0}$.

There exist $\varepsilon \in(0,1), t>0$ such that for all $\delta \in(0,1), s>0$ there exist $f \in F(X)$ and $N\left(f-f_{0}, s\right)>1-$ $\delta \Rightarrow N\left(\Psi(f)-\Psi\left(f_{0}\right) t\right) \leq 1-\varepsilon \Rightarrow$ for all $n \in Z^{+}$there exist $f_{n} \in F(X)$ such that

$N\left(f_{n}-f_{0}, s\right)>1-\frac{1}{n} \Rightarrow N\left(\psi\left(f_{n}\right)-\psi\left(f_{0}\right), t\right) \leq 1-\varepsilon$ that is mean $f_{n} \rightarrow f_{0}$ in $F(X)$, but $\psi\left(f_{n}\right) \rightarrow \psi\left(f_{0}\right)$ in $Y$ this contradiction, $\psi$ is fuzzy continuous at $f_{0}$.

Theorem3.11. Let $\left(F(X), N_{1}, *\right)\left(F(Y), N_{2}, *\right)$ be two-fuzzy normed spaces. If the functions $\Psi: F(X) \rightarrow F(Y)$, $\omega:(X) \rightarrow F(Y)$ are two fuzzy continuous functions and with for all $a$ there exist $a_{1}$ such that $a_{1} * a_{1} \geq$ $a$ and $a, a_{1} \in(0,1)$ then:

(1) $f+g,(2) k f$ where $k \in \mathbb{F} /\{0\}$, are also fuzzy continuous functions over the same filed $\mathbb{F}$.

Proof: (1)Let $\varepsilon \in(0,1)$ then there exists $\varepsilon_{1} \in(0,1)$ such that $\quad\left(1-\varepsilon_{1}\right) *\left(1-\varepsilon_{1}\right) \geq(1-\varepsilon)$.

Let $\left\{f_{n}\right\}$ be a sequence in $F(X)$ such that $f_{n} \rightarrow f$. Since $\psi, \omega$ are two fuzzy continuous functions at $f$ thus for all $\varepsilon_{1} \in(0,1)$ and all $t>0$ there exist $\delta \in(0,1)$ and $s>0$ such that for all $f \in F(X): N_{1}\left(f_{n}-f, s\right)>1-\delta$ implies $N_{2}\left(\psi\left(f_{n}\right)-\psi(f), \frac{t}{2}\right)>1-\varepsilon_{1}$.

And $N_{1}\left(f_{n}-f, s\right)>1-\delta$ implies $N_{2}\left(\omega\left(f_{n}\right)-\omega(f), \frac{t}{2}\right)>1-\varepsilon_{1}$

Now: $\quad N_{2}\left((\Psi+\omega)\left(f_{n}\right)-(\Psi+\omega)(f), t\right)=N_{2}\left(\Psi\left(f_{n}\right)+\omega\left(f_{n}\right)-\psi(f)-\omega(f), t\right) \geq N_{2}\left(\psi\left(f_{n}\right)-\psi(f), \frac{t}{2}\right) *$

$N_{2}\left(\omega\left(f_{n}\right)-\omega(f), \frac{t}{2}\right)$

Then $\psi+\omega$ is fuzzy continuous function.

$$
>\left(1-\varepsilon_{1}\right) *\left(1-\varepsilon_{1}\right) \geq(1-\varepsilon)
$$

(2) Let $\left\{f_{n}\right\}$ be a sequence in $F(X)$ such that $f_{n} \rightarrow f$.Thus for all $\varepsilon_{1} \in(0,1)$ and for all $t>0$, there exist $\delta \in$ $(0,1)$ and $s>0 \ni N_{1}\left(f_{n}-f, s\right)>1-\delta$ implies $N_{2}\left(\psi\left(f_{n}\right)-\psi(f), t\right)>1-\varepsilon_{1}$, take $t_{1}=t|k|$.

Then for all $\varepsilon_{1} \in(0,1)$ and for all $t_{1}>0$, there exist $\delta \in(0,1)$ and $s>0 \ni N_{1}\left(f_{n}-f, s\right)>1-\delta$ implies

$$
\begin{gathered}
\mathrm{N}_{2}\left((k \Psi)\left(f_{n}\right)-(k \Psi)(f), t_{1}\right)=N_{2}\left(k\left(\psi\left(f_{n}\right)-\psi(f)\right), t_{1}\right) \\
=N_{2}\left(\Psi\left(f_{n}\right)-\psi(f), t\right)>1-\varepsilon_{1}
\end{gathered}
$$

Thenk $\psi$ is a fuzzy continuous function.

\section{References}

[1]. J. Zhang, The continuity and boundedness of fuzzy linear operators in fuzzy normed space, J.Fuzzy Math. 13(3) (2005) 519-536.

[2]. L. A. Zadeh, Fuzzy sets, Information and Control 8 (1965) 338-353.

[3]. RM. Somasundaram and ThangarajBeaula, Some Aspects of 2-fuzzy 2-normed linear spaces, Bull. Malays. Math. Sci. Soc. 32(2) (2009) 211-222.

[4]. S. Gahler, Lineare 2-normierte Raume, Math. Nachr. 28 (1964) 1- 43.

[5]. ThangarajBeaula, R. Angeline SargunaGifta. Some aspects of 2-fuzzy inner product space. Annals of Fuzzy Mathematics and Informatics Volume 4, No. 2, (October 2012), pp. 335-342

[6]. T. Bag and S. K. Samanta, Finite dimensional fuzzy normed linear spaces, J. Fuzzy Math. 11(3) (2003) 687-705. 